\title{
MiR-19a mediates gluconeogenesis by targeting PTEN in hepatocytes
}

\author{
LIN DOU, SHUYUE WANG, XIUQING HUANG, XUELIN SUN, YANG ZHANG, \\ TAO SHEN, JUN GUO, YONG MAN, WEIQING TANG and JIAN LI
}

The MOH Key Laboratory of Geriatrics, Beijing Hospital, National Center of Gerontology, Beijing 100730, P.R. China

Received August 24, 2017; Accepted December 14, 2017

DOI: $10.3892 / \mathrm{mmr} .2017 .8312$

\begin{abstract}
As a member of miR-17-92 miRNA clusters, miR-19a has been considered to regulate hepatic glycogenesis by mediating the PI3K/AKT signaling pathway. However, whether miR-19a serves an important role in gluconeogenesis in hepatocytes remains unknown. In the present study, the impact of miR-19a on gluconeogenesis in HEP1-6 cells and its mechanisms of action were investigated. It was observed that overexpression of miR-19a led to decreased glucose production, accompanied by increased activation of the AKT/FOXO1 signaling pathway and downregulated expression of gluconeogenesis-associated genes, including peroxisome proliferator-activated receptor $\gamma$ coactivator $1 \alpha$, phosphoenolpyruvate carboxykinase and glucose 6-phosphatase in the HEP1-6 cells transfected with the miR-19a mimic. In contrast, suppression of miR-19a impaired the activation of the AKT/FOXO1 signaling pathway and increased the expression of gluconeogenesis-associated genes, accompanied by an elevated glucose production. Additionally, phosphatase and tensin homolog (PTEN) was identified as a target of miR-19a and participated in the miR-19a-mediated gluconeogenesis in hepatocytes. These findings provide mechanistic insight into the effects of miR-19a on the regulation of the AKT/FOXO1 signaling pathway and the expression of gluconeogenesis-associated genes. MiR-19a may mediate gluconeogenesis in hepatocytes by downregulating PTEN expression.
\end{abstract}

\section{Introduction}

The dysregulation of glucose homeostasis is a critical cause for type 2 diabetes mellitus (1). Decreased insulin secretion and insulin sensitivity is hall marks of type 2 diabetes

Correspondence to: Dr Jian Li, The MOH Key Laboratory of Geriatrics, Beijing Hospital, National Center of Gerontology, 1 Dahua Road, Beijing 100730, P.R. China

E-mail: lijian@bjhmoh.cn

Key words: miR-19a, gluconeogenesis, PTEN, FOXO1, PGC1 $\alpha$, PEPCK, G6Pase mellitus (2). Insulin secreted by pancreas can decrease glucose production and increase uptake of blood glucose into glycogen in liver. Therefore, hepatic insulin resistance leads to increased glucose production and decreased glycogenesis, resulting in elevated blood glucose levels (3). MicroRNAs are a cluster of endogenous small non-coding RNAs, which can negatively regulate genes expression at the post-transcriptional level, either by inhibiting translation or by degrading the target mRNAs (4). In our previous study, we indicated many miRNAs involved in regulation of glycogenesis in hepatocytes, such as miR-200s (5), miR-301a (6), miR-152-3p (7) and miR-20a-5p (8). Moreover, we found an important role of miR-19a in regulating of glycogenesis in hepatocytes (9). It was reported that miR-19a is a member of miR-17-92 family, which is located on chromosome $13 \mathrm{q} 31.3$ and is related with the pathogenesis of cancer (10). MiR-19a could affect the activation of PI3K/AKT pathway by targeting phosphatase and tensin homolog (PTEN) in mouse liver cells. However, whether miR-19a serves an important role in glucose production in hepatocytes remains unknown.

Here, we define the impact of miR-19a on gluconeogenesis and its underlying mechanisms. Our findings suggest that miR-19a plays an important role in gluconeogenesis via targeting PTEN to regulate the activation of AKT/FOXO1 pathway.

\section{Methods and materials}

Cell culture. The HEP1-6 murine liver cell line (American Type Culture Collection) was cultured in low-glucose Dulbecco's modified Eagle's medium (L-DMEM; Invitrogen Life Technologies, Carlsbad, CA, USA) supplemented with $10 \%$ fetal serum (Hyclone; GE Healthcare Life Sciences, Logan, UT, USA), 100 units/ml penicillin (Invitrogen Life Technologies), and $0.1 \mathrm{mg} / \mathrm{ml}$ streptomycin (Hyclone) at $37^{\circ} \mathrm{C}$ with humidified air and $5 \% \mathrm{CO}_{2}$.

Transfection of miR-19a mimic and inhibitor. The sequences of miR-19a mimic and inhibitor were as follows (5'-3'): miR-19a mimic sense, UGUGCAAAUCUAUGCAAAACUGA and antisense, AGUUUUGCAUAGAUUUGCACAUU; miR-19a inhibitor, UCAGUUUUGCAUAGAUUUGCACA.MicroRNA oligos were purchased from Genepharma (Shanghai, China). According to the manufacturer's instruction, negative miRNA 
mimic control (NC), miR-19a mimic (19am), negative miRNA inhibitor control (NCi) and miRNA-19a inhibitor (19ai) were transfected into HEP1-6 cells by using Hiperfect transfection reagent (Qiagen, Hilden, Germany). Before transfection, seeded HEP1-6 cells in 6-well plate at $1.0 \times 10^{5}$ cell per well. Diluted $37.5 \mathrm{ng}$ miRNA and $3 \mu \mathrm{l}$ Hiperfect transfection reagent in $100 \mu \mathrm{l} \mathrm{L}-\mathrm{DMEM}$ and mixed by vortexing. The mixture was added into the cell culture medium and incubated for $48 \mathrm{~h}$.

Quantification of miR-19a expression by reverse transcription-quantified polymerase chain reaction. Total RNA was harvested using TRIzol (Invitrogen Life Technologies) after transfection for $48 \mathrm{~h}$. Quantification of miR-19a levels was using by real-time PCR according the protocol of SYBR-Green II kit (Takara Bio, Inc., Otsu, Japan). The sequences of reverse transcription primers were as follows: (5'-3') miR-19a, GTCGTATCCAGTGCAGGGTCCGAGGTA TTCGCACTGGATACGACTCAGTT; U6, GTCGTATCC AGTGCAGGGTCCGAGGTATTCGCACTGGATACGACA

AAAATATG. The nucleotide primers used for real-time PCR were as follows (5'-3'): miR-19a forward, GCGTGTGCAAAT CTATGCAA; U6 forward, GCGCGTCGTGAAGCGTTC; universal reverse primer, GTGCAGGGTCCGAGGT.

Western blot. After transfection for $48 \mathrm{~h}$, cell lysates (15 $\mu \mathrm{g}$ of protein) were harvest and separated by $10 \%$ SDS-PAGE, transferred to PVDF membranes (EMD Millipore, Billerica, MA, USA), blocked with $8 \%$ nonfat dry milk, and probed with 1:1,000 primary antibodies at $4^{\circ} \mathrm{C}$ overnight. The blots were incubated with 1:5,000 HRP-conjugated anti-IgG for $1 \mathrm{~h}$ at room temperature, followed by detection with ECL (EMD Millipore). The antibodies against AKT (9272S), phosphorylated AKT (ser473) (4060S), FOXO1 (2880), phosphorylated FOXO1 (ser256) (9461), PEPCK (6924) and GAPDH (5174) were purchased from Cell Signaling Technology, Inc. (Danvers, MA, USA). The antibodies against PGC1 $\alpha$ (ab54481) and G6Pase (ab83690) were purchased from Abcam (Cambridge, UK).

Immunofluorescence. HEP1-6 cells were seed on coverslips and fixed with $4 \%$ paraformaldehyde for $20 \mathrm{~min}$ at room temperature. Washed the slides with PBS for three times, and permeabilized with $0.2 \%$ Triton X-100/PBS for $10 \mathrm{~min}$ at room temperature. Then blocked with 3\% BSA/PBS for $20 \mathrm{~min}$ at room temperature and incubated with 1:300 primary antibody against $\mathrm{FOXO1}$ over night at $4^{\circ} \mathrm{C}$. The slides were washed three times with PBS and incubated with 1:100 secondary antibody for $1 \mathrm{~h}$ at $37^{\circ} \mathrm{C}$. To stain cellular nuclear, the cells were incubated with $10 \mathrm{mM}$ Hoechest 333442 to stain DNA. Then the coverslips were mounted in glycerol. The cells were analyzed by using a ZEISS LSM700 immunofluorescence microscope. The images were captured with $\mathrm{CCD}$ and Axiovision image software.

Glucose production assay. The cells were washed five times with PBS and the stimulated with $2 \mathrm{mmol} / \mathrm{l}$ sodium pyruvate and $20 \mathrm{mmol} / \mathrm{l}$ sodium lactate in glucose- and serum-free DMEM medium for $18 \mathrm{~h}$. The glucose concentration in the medium was analyzed by using a glucose assay kit (Sigma) and normalized to the total protein content determined from the whole cell extracts.
Statistical analysis. All data were presented as mean \pm SEM. The two-tailed unpaired student's t-test was used for comparisons of two groups. The ANOVA multiple comparison test (SPSS 3.0; SPSS, Inc., Chicago, IL, USA) followed by Turkey post hoc test were used for comparisons of two more groups. $\mathrm{P}<0.05$ was considered to indicate a statistically significant difference.

\section{Results}

Upregulation of miR-19aleadstodecreased glucose production and expression of gluconeogenetic genes, increased level of p-AKT and p-FOXOI in HEPI- 6 cells. In our previous study, we found that miR-19a elevated the activation of AKT/GSK pathway and the glycogenesis in mouse hepatic cells. However, the effects of miR-19a on gluconeogenesis in hepatic cells still unknown. To determine the effects of miR-19a on the glucose production and the expression of gluconeogenetic genes including PGC-1 $\alpha$, G6Pase and PEPCK, miR-19a mimic was transfected into the HEP1-6 cells. As shown in Fig. 1A, the level of miR-19a was increased to almost 50-fold in HEP1-6 cells transfected with miR-19a mimic. Moreover, over-expression of miR-19a suppressed the expression of PGC-1 $\alpha$, G6Pase and PEPCK, accompanied by elevated levels of p-AKT and p-FOXO1 in the HEP1-6 cells transfected with miR-19a mimic (Fig. 1B). The confocal analysis showed that FOXO1 located in cytoplasm in HEP1-6 cells transfected with miR-19a mimic (Fig. 1C). The gluconeogenesis level was decreased in HEP1-6 cells transfected with miR-19a mimic (Fig. 1D). Taken together, Upregulation of miR-19a impaired glucose production by downregulating expression of gluconeogenetic genes and stimulating activation AKT/FOXO1 pathway in HEP1-6 cells.

Downregulation of miR-19a promotes glucose production and expression of gluconeogenetic genes and suppresses activation of AKT/FOXO1 pathway in HEPI-6 cells. To gain further insight into the significance of miR-19a in regulating gluconeogenesis, miR-19a inhibitor was transfected into HEP1-6 cells. The level of miR-19a was decreased to 50\% in HEP1-6 cells transfected with miR-19a inhibitor (Fig. 2A). Importantly, the expression of PGC-1 $\alpha$, G6Pase and PEPCK was enhanced significantly, while activation of AKT/FOXO1 pathway was suppressed in HEP1-6 cells transfected with miR-19a inhibitor (Fig. 2B). FOXO1 located in nuclear in HEP1-6 cells transfected with miR-19a inhibitor (Fig. 2C). The glucose production was increased in HEP1-6 cells transfected with miR-19a inhibitor (Fig. 2D). These results suggest that downregulation of miR-19a promoted glucose production through blocking activation of AKT/FOXO1 signaling and increasing expression of gluconeogenetic genes in HEP1-6 cells.

Silence of PTEN impairs glucose production and expression of gluconeogenetic genes. In our previous study, we found that PTEN is a target gene of miR-19a. And PTEN could regulate activation of PI3K/AKT pathway. To explore the effect of PTEN on glucose production and expression of gluconeogenetic genes, a siRNA specifically targeted PTEN (si-1519) was transfected into HEP1- 6 cells for $48 \mathrm{~h}$ (9). The protein level of PTEN was decreased significantly (Fig. 3A). And the 
A

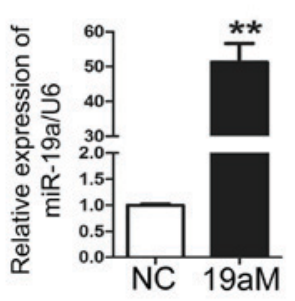

B

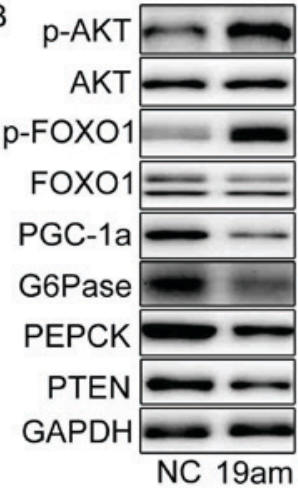

DAPI

C

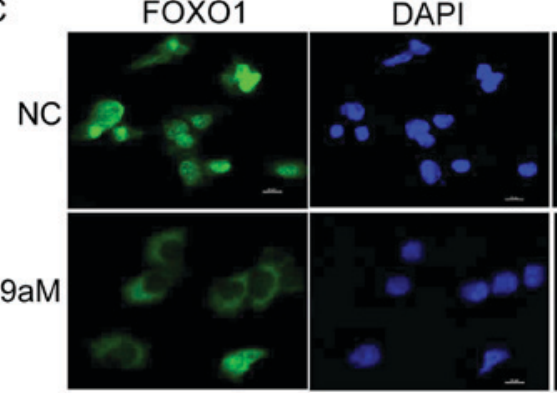

Merged

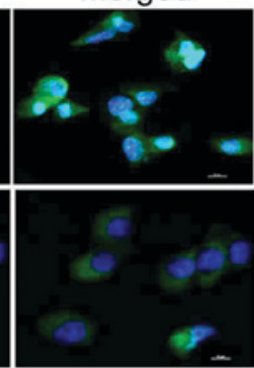

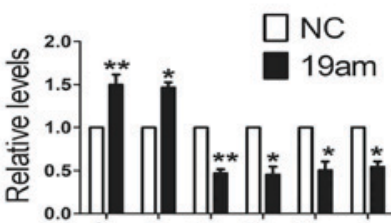

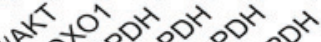

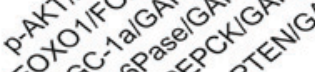

Figure 1. Upregulation of miR-19a led to decreased glucose production and expression of gluconeogenetic genes, increased level of p-AKT and p-FOXO1 in HEP1-6 cells. Negative miRNA mimic control and miR-19a mimic were transfected into HEP1-6 cells for $48 \mathrm{~h}$. Compared with the cells transfected with negative miRNA mimic control (NC), the level of miR-19a was increased to almost 50-fold in HEP1-6 cells transfected with miR-19a mimic (19aM) (A) Over-expression of miR-19a suppressed expression of PGC-1 $\alpha$, G6Pase and PEPCK, accompanied by elevated levels of p-AKT and p-FOXO1 (B) in the HEP1-6 cells transfected with miR-19a mimic. The confocal analysis of that FOXO1 located in cytoplasm in HEP1-6 cells transfected with miR-19a mimic (C) The gluconeogenesis level was decreased in HEP1-6 cells transfected with miR-19a mimic (D). N=4 independent experiments. "P<0.05; ${ }^{* *} \mathrm{P}<0.01$ (vs. control).
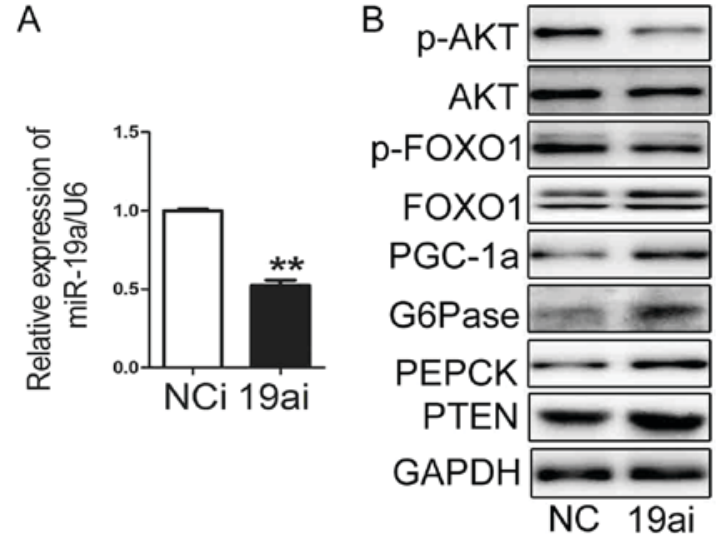

C

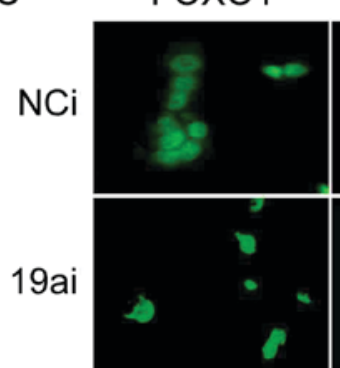

DAPI

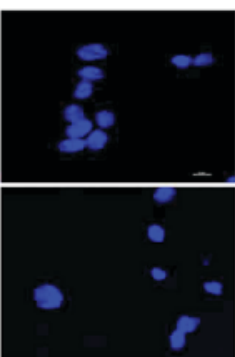

Merged

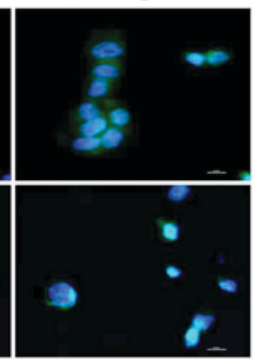

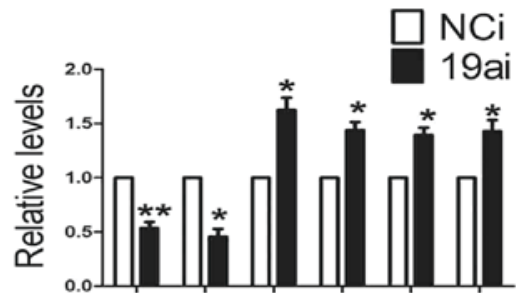

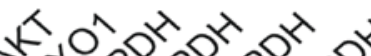

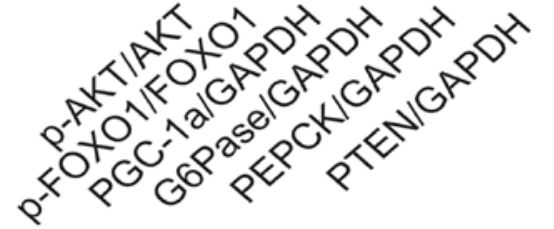

D

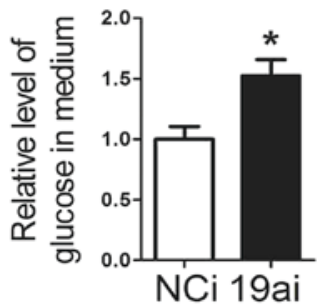

Figure 2. Downregulation of miR-19a promoted glucose production and expression of gluconeogenetic genes and suppressed activation of AKT/FOXO1 pathway in HEP1-6 cells. Negative miRNA inhibitor control and miR-19a inhibitor were transfected into HEP1-6 cells for 48 h. Compared with the cells transfected with negative miRNA inhibitor control (NCi), the level of miR-19a was decreased to 50\% in HEP1-6 cells transfected with miR-19a inhibitor (19ai) (A), The expression of PGC-1 $\alpha$, G6Pase and PEPCK (B) was enhanced significantly, while activation of AKT/FOXO1 pathway (B) was suppressed in HEP1-6 cells transfected with miR-19a inhibitor. The confocal analysis showed that FOXO1 located in nuclear in HEP1-6 cells transfected with miR-19a inhibitor (C). The gluconeogenesis level was increased in HEP1-6 cells transfected with miR-19a mimic (D). N=4 independent experiments. ${ }^{*} \mathrm{P}<0.05$; ${ }^{* *} \mathrm{P}<0.01$ (vs. control). 

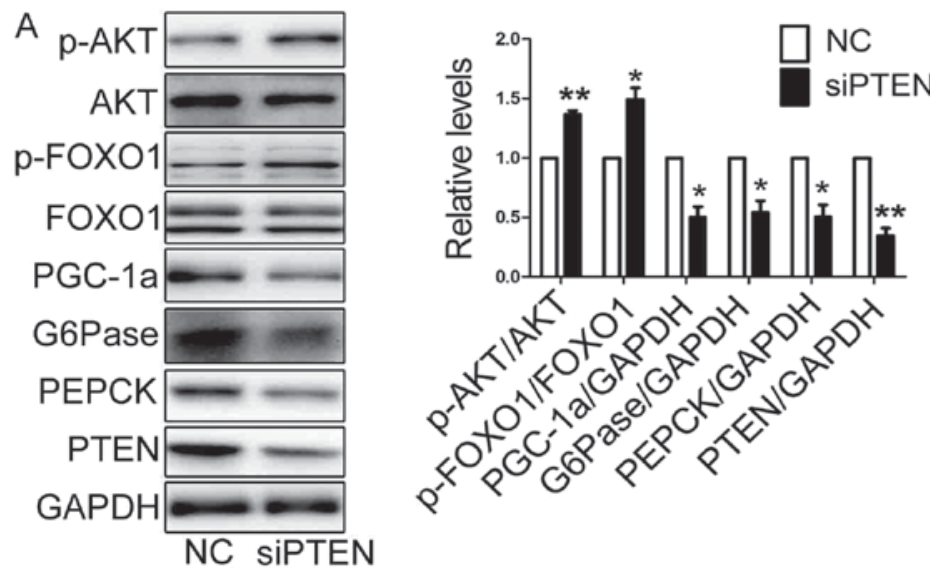

B

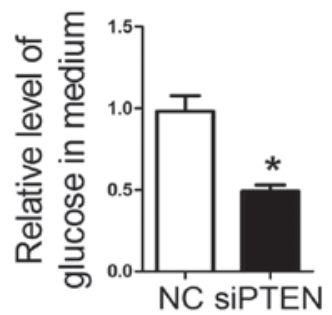

Figure 3. Silence of PTEN impaired glucose production and expression of gluconeogenetic genes. A siRNA specifically targeted PTEN (siPTEN) was transfected into HEP1-6 cells for $48 \mathrm{~h}$. Compared with the cells transfected with negative siRNA control (NC), the expression of PGC-1 $\alpha$, G6Pase and PEPCK were reduced, while activation of AKT/FOXO1 pathway was elevated in HEP1-6 cells transfected with si-PTEN in HEP1-6 cells transfected with si-PTEN (si-PTEN) (A). The glucose production was decreased in HEP1-6 cells transfected with si-PTEN (B). N=4 independent experiments. "P<0.05; ${ }^{* *} \mathrm{P}<0.01$ (vs. control).
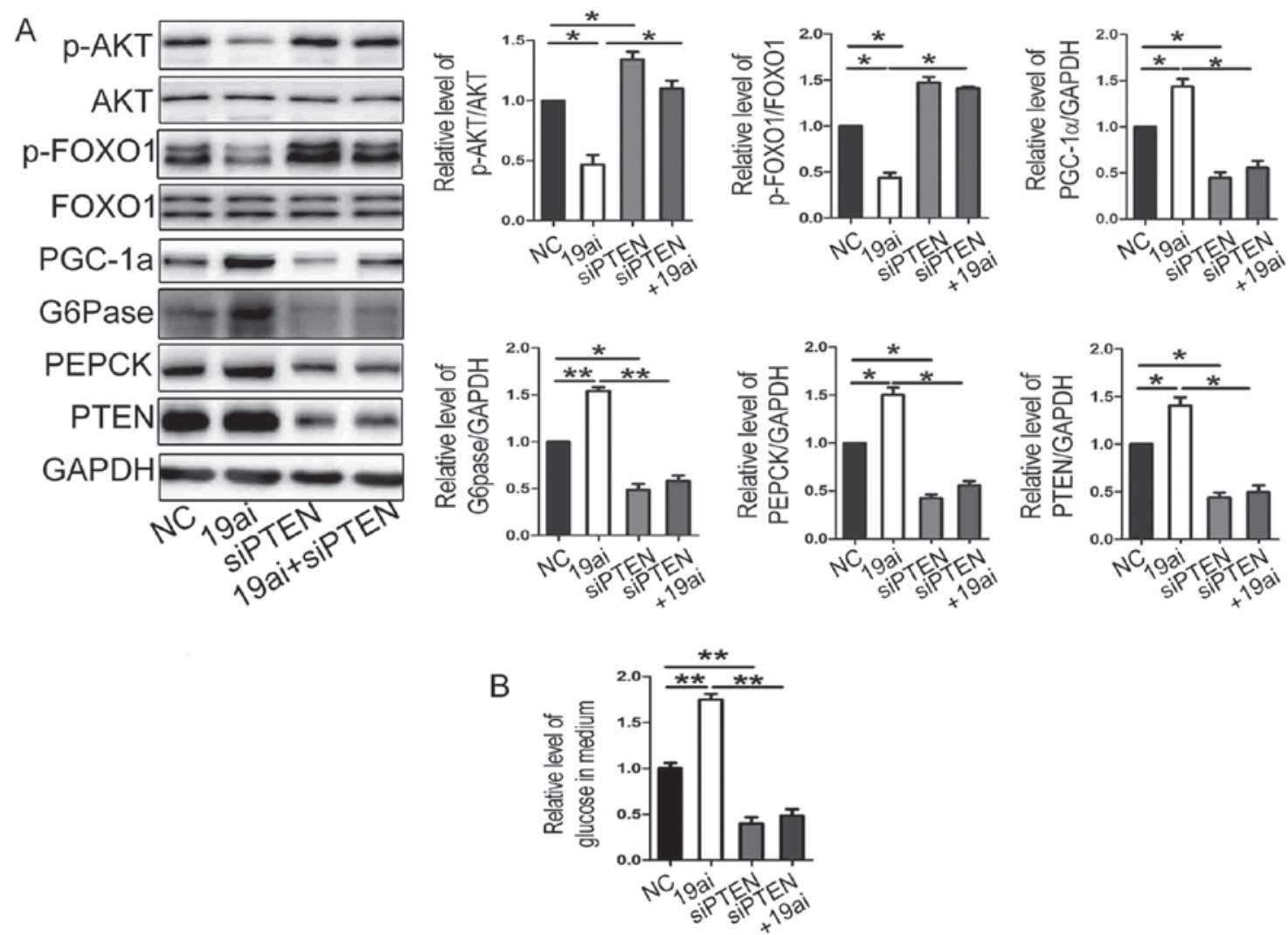

Figure 4. MiR-19a regulates glucose production and expression of gluconeogenetic genes via targeting PTEN. The miR-19a inhibitor and siPTEN were co-transfected into HEP1-6 cells. Transfection of siPTEN reversed the effect of miR-19a inhibitor on expression of gluconeogenetic genes (A) and glucose production (B) in HEP1- 6 cells. $\mathrm{N}=4$ independent experiments. ${ }^{*} \mathrm{P}<0.05 ;{ }^{* *} \mathrm{P}<0.01$ (vs. control).

expression of PGC-1 $\alpha$, G6Pase and PEPCK were reduced, while the activation of AKT/FOXO1 pathway were elevated in HEP1-6 cells transfected with siPTEN (Fig. 3A). The glucose production was decreased in HEP1-6 cells transfected with siPTEN (Fig. 3B).

MiR-19a regulates glucose production and expression of gluconeogenetic genes via targeting PTEN. Next, to verify that
miR-19 regulates glucose production and expression of gluconeogenetic genes via targeting PTEN, the miR-19a inhibitor and siPTEN were co-transfected into HEP1-6 cells. As shown in Fig. 4 that transfection of siPTEN reversed the effect of miR-19a inhibitor on expression of gluconeogenetic genes (Fig. 4A) and glucose production (Fig. 4B). Taken together, miR-19a might regulate glucose production and expression of gluconeogenetic genes and via targeting PTEN. 


\section{Discussion}

The impaired hepatic insulin sensitivity contributes to increased hepatic glucose production, which may lead to dysregulation of glucose metabolism $(11,12)$. There increasing evidence that miRNAs are involved in the regulation of glucose metabolism (4). MiR-19a, a member of mir-17-92 miRNA family, is a oncogenic miRNA, which could promote proliferation and angiogenesis of cancer cell (10). In our previous study, we verified that over-expression of miR-19a increased the activation of PI3K/AKT/GSK pathway and glycogenesis via targeting PTEN. In the present study, we focused on the critical role of miR-19a in regulating hepatic glucose production. Our results suggested that over-expression of miR-19a elevated the phosphorylation of FOXO1 and suppressed expression of gluconeogenesis- related genes, such as PGC1 $\alpha$, PEPCK and G6Pase. Most importantly, glucose production was impaired in HEP1-6 cells transfected with miR-19a mimic. It was reported that PI3K/AKT pathway is the main insulin signal pathway in hepatocytes, which can regulate hepatic glucose metabolism including gluconeogenesis and glycogenesis (1). Activated AKT can inactivate glycogen synthase kinase $3 \beta$ (GSK3 $\beta$ ), which permits the activation of glycogen synthase and leads to glycogenesis $(13,14)$. Moreover, activated AKT also can promote the phosphorylation and nuclear exclusion of FOXO1 to lower expression of gluconeogenetic enzymes and gluconeogenesis (15). In hepatic insulin resistance, suppressed activation of PI3K/AKT led to increased hepatic gluconeogenesis through reduced inactivation of FOXO1. Furthermore, PGC1 $\alpha$ is another transcriptional co-activator which is activated under fasting conditions (16). PGC1 $\alpha$ co-activates FOXO1, leading to PEPCK and G6Pase transcription. Insulin impaired PGC1 $\alpha$ by increasing its phosphorylation at ser570 by activated AKT (17).

In previous study, PTEN was verified as a direct target of miR-19a to mediate the activation of PI3K/AKT/GSK pathway. There are several binding sites for miR-19a at the PTEN 3'-UTR. PTEN is expressed in all tissues and contains a tensin-like domain and a phosphatase catalytic domain $(18,19)$. Moreover, PTEN is a negative regulator of PI3K/AKT pathway by catalyzing PIP3 dephosphorylation and converting it into PIP2 (19-21). In the present study, we found that PTEN could regulate phosphorylation of FOXO1. Silencing PTEN could reverse the effects of miR-19a inhibition on phosphorylation of FOXO1 and expression of gluconeogenesis- related genes. Therefore, PTEN participated in miR-19a-mediated gluconeogenesis in hepatocytes via regulating AKT/FOXO1 pathway.

In conclusion, these findings provide mechanistic insight into the effects of miR-19a on gluconeogenesis and regulation of AKT/FOXO1 pathway in hepatocytes. MiR-19a might mediate gluconeogenesis via downregulating PTEN expression.

\section{Acknowledgements}

The present study was supported by grants (81570789 and 81600618) from National Natural Science Foundation of China.

\section{References}

1. Rines AK, Sharabi K, Tavares CD and Puigserver P: Targeting hepatic glucose metabolism in the treatment of type 2 diabetes. Nat Rev Drug Discov 15: 786-804, 2016.
2. Bugianesi E, McCullough AJ and Marchesini G: Insulin resistance: A metabolic pathway to chronic liver disease. Hepatology 42: 987-1000, 2005.

3. Leclercq IA, Da Silva Morais A, Schroyen B, Van Hul N and Geerts A: Insulin resistance in hepatocytes and sinusoidal liver cells: Mechanisms and consequences. J Hepatol 47: 142-156, 2007.

4. Fernandez-Valverde SL, Taft RJ and Mattick JS: MicroRNAs in beta-cell biology, insulin resistance, diabetes and its complications. Diabetes 60: 1825-1831, 2011.

5. Dou L, Zhao T, Wang L, Huang X, Jiao J, Gao D, Zhang H, Shen T, Man Y, Wang S and Li J: miR-200s contribute to interleukin-6 (IL-6)-induced insulin resistance in hepatocytes. J Biol Chem 288: 22596-22606, 2013

6. Dou L, Wang S, Sui X, Meng X, Shen T, Huang X, Guo J, Fang W, Man Y, Xi J and Li J: MiR-301a mediates the effect of IL-6 on the AKT/GSK pathway and hepatic glycogenesis by regulating PTEN expression. Cell Physiol Biochem 35: 1413-1424, 2015.

7. Wang S, Wang L, Dou L, Guo J, Fang W, Li M, Meng X, Man Y, Shen T, Huang X and Li J: MicroRNA 152 regulates hepatic glycogenesis by targeting PTEN. FEBS J 283: 1935-1946, 2016.

8. Fang W, Guo J, Cao Y, Wang S, Pang C, Li M, Dou L, Man Y, Huang X, Shen T and Li J: MicroRNA-20a-5p contributes to hepatic glycogen synthesis through targeting p63 to regulate p53 and PTEN expression. J Cell Mol Med 20: 1467-1480, 2016.

9. Dou L, Meng X, Sui X, Wang S, Shen T, Huang X, Guo J, Fang W, Man Y, Xi J and Li J: MiR-19a regulates PTEN expression to mediate glycogen synthesis in hepatocytes. Sci Rep 5: 11602, 2015.

10. Chen L, Li C, Zhang R, Gao X, Qu X, Zhao M, Qiao C, Xu J and Li J: miR-17-92 cluster microRNAs confers tumorigenicity in multiple myeloma. Cancer Lett 309: 62-70, 2011.

11. Lanthier $\mathrm{N}$ and Leclercq IA: Liver and systemic insulin resistance. Hepatology 60: 1113-1114, 2014.

12. Meshkani R and Adeli K: Hepatic insulin resistance, metabolic syndrome and cardiovascular disease. Clin Biochem 42: 1331-1346, 2009.

13. Samue VT and Shulman GI: The pathogenesis of insulin resistance: Integrating signaling pathways and substrate flux. J Clin Invest 126: 12-22, 2016

14. Wan M, Leavens KF, Hunter RW, Koren S, von Wilamowitz-Moellendorff A, Lu M, Satapati S, Chu Q, Sakamoto K, Burgess SC and Birnbaum MJ: A noncanonical, GSK3-independent pathway controls postprandial hepatic glycogen deposition. Cell Metab 18: 99-105, 2013.

15. Lu M, Wan M, Leavens KF, Chu Q, Monks BR, Fernandez S, Ahima RS, Ueki K, Kahn CR and Birnbaum MJ: Insulin regulates liver metabolism in vivo in the absence of hepatic Akt and Foxol. Nat Med 18: 388-395, 2012.

16. Yoon JC, Puigserver P, Chen G, Donovan J, Wu Z, Rhee J, Adelmant G, Stafford J, Kahn CR, Granner DK, et al: Control of hepatic gluconeogenesis through the transcriptional coactivator PGC-1. Nature 413: 131-138, 2001.

17. Puigserver P, Rhee J, Donovan J, Walkey CJ, Yoon JC, Oriente F, Kitamura Y, Altomonte J, Dong H, Accili D and Spiegelman BM: Insulin-regulated hepatic gluconeogenesis through FOXO1-PGC-1alpha interaction. Nature 423: 550-555, 2003.

18. Gupta A and Dey CS: PTEN, a widely known negative regulator of insulin/PI3K signaling, positively regulates neuronal insulin resistance. Mol Biol Cell 23: 3882-3898, 2012.

19. Horie Y, Suzuki A, Kataoka E, Sasaki T, Hamada K, Sasaki J, Mizuno K, Hasegawa G, Kishimoto H, Iizuka M, et al: Hepatocyte-specific Pten deficiency results in steatohepatitis and hepatocellular carcinomas. J Clin Invest 113: 1774-1783, 2004.

20. Guenzl PM, Raim R, Kral J, Brunner J, Sahin E and Schabbauer G: Insulin hypersensitivity induced by hepatic PTEN gene ablation protects from murine endotoxemia. PLoS One 8: e67013, 2013.

21. Matsuda S, Kobayashi M and Kitagishi Y: Roles for $\mathrm{PI} 3 \mathrm{~K} / \mathrm{AKT} / \mathrm{PTEN}$ pathway in cell signaling of nonalcoholic fatty liver disease. ISRN endocrinol 2013: 472432, 2013. 